\title{
Experimental control programs for the UNIX operating system
}

\author{
GARY PERLMAN \\ University of California at San Diego, La Jolla, California 92093
}

\begin{abstract}
The software described runs on Version 7 UNIX, although most run on earlier versions. Using the curses package requires two program libraries from the Fourth Berkeley Distribution (CURSES and TERMLIB). Using any of the functions requires knowledge of programming, preferably in C (Kernighan \& Richie, 1978), but the sample experimental control program is usable by nonprogrammers. Because no special hardware is assumed, the resolution of timed presentations and reaction time recordings may not be accurate enough for some purposes. Functions for stimulus presentation have a resolution of $1 \mathrm{sec}$, and those for timing responses are accurate to about $17 \mathrm{msec}$.
\end{abstract}

This paper may serve as an organizer and reference for existing software useful for programming psychological experiments on the UNIX operating system. UNIX offers hundreds of system calls that have varying applicability to experimental control programming. Many of these system cells are hard to find and, even if they are found, the usefulness of a function is not always apparent. In this paper, I give examples of their use.

This paper also offers some software tools for writing programs for controlling experiments. While the software UNIX offers is useful, it is not specifically suited to the needs of psychology experimenters. Some higher level functions, however, make use of low-level functions supplied by UNIX.

The paper serves as a tutorial on writing experimental control programs. Concrete examples showing where and how software tools can be used are necessary if they are to be used at all.

The software is written in C (Kernighan \& Richie, 1978), a structured algorithmic language similar to ALGOL and PASCAL. C is the systems programming language of the UNIX operating system (Richie \& Thompson, 1974, 1978), developed at Bell Laboratories and licensed by Western Electric. The University of California at Berkeley (UCB; Joy, 1980) releases an

I thank Mark Wallen for his help with UNIX programming and Tom Erickson for his helpful comments. Jay McClelland wrote the first version of PERM. This work was completed while I was supported by a postgraduate scholarship from the Natural Science and Engineering Research Council of Canada. This research was conducted under Contract N00014-79-C-0323, NR 157437, with the Personnel and Training Research Programs of the Office of Naval Research, and was sponsored by the Office of Naval Research and the Air Force Office of Scientific Research. Copies of software and supporting documentation can be obtained by writing Gary Perlman, Department of Psychology, C009, University of California at San Diego, La Jolla, California 92093. enhanced version of UNIX that includes software described in this paper. While the functions are written in C, UNIX allows programs written in FORTRAN 77 (Katzan, 1978) and PASCAL (Jensen \& Wirth, 1974) to be used in function calls.

UNIX has become popular in the experimental psychology community because of its availability for DEC PDP-11 and VAX computers ${ }^{1}$ and because of its versatility for program development and text processing. One use of UNIX is the control of psychological experiments; many experiments can be run using UNIXsupplied functions without special hardware. Stimulus randomization, stimulus presentation at a specified location on a screen, and collection of timed responses are all possible with a character CRT terminal.

This paper is an organizer and reference to functions useful for experimental control developed at Bell, UCB, and the Cognitive Science Laboratory (CSL) at the University of California at San Diego (UCSD). The sources of functions are mentioned as they are introduced and can be referred to in the appendix. The structure of the first part of the paper follows that of an experimental control program. Functions are discussed for (1) stimulus randomization, (2) for stimulus presentation, and (3) for timed response collection. In the second part of the paper, a general experimental control program that uses all the functions is described. It is practically useful, as it can run a variety of paradigms. The control program also serves as a model for writing new programs; for that reason, in the second part of this paper I discuss practical considerations in writing experimental control programs.

Functions used within $C$ programs are called system calls or utility functions. These are described in sections 2 and 3 of the UNIX programmer's manual (Joy, 1980) if they are from Bell Laboratories or UCB, or in documentation available from the author. In a few cases, reference is made to UNIX level programs, which 
are used at the same level as commands for text editing or reading mail. (The convention is to capitalize the names of UNIX programs and systems programming functions, although in practice, they would be lowercase.)

\section{EXPERIMENTAL CONTROL FUNCTIONS}

The basic components of an experimental control program are: stimulus randomization, stimulus presentation, and response collection. (Although the functions available and examples are provided in this discussion, the UNIX programmer's manual should be used as the final reference.)

\section{STIMULUS RANDOMIZATION}

Two types of stimulus randomization are considered: randomization of stimulus files before the experiment begins and randomization of stimuli stored inside an experimental control program. I will assume each stimulus occupies one line in a stimulus file. If each stimulus is presented just once, experimental programs can be made more compact because stimuli can be randomized outside the program and only one stimulus need be stored at any time. Internal randomization allows more freedom but requires more stimulus storage space.

\section{File Randomization}

PERM is a CSL program for randomizing stimulus files that reads in a file and prints the file's lines in random order. PERM is used at the UNIX command level, and so can be used without programming expertise. For example, if stimuli are stored in a file called "stim," the command

\section{PERM stim mixed}

puts a random ordering of stim's lines in "mixed." The lines in this randomized file can then be presented in order, since the order is random.

\section{File Randomization from Inside a Program}

$C$ provides a function to execute UNIX-level commands from inside programs. The SYSTEM function executes the string COMMAND as though it were typed at the UNIX command level:

$$
\text { system(command); }
$$

This function can be used for randomizing stimulus files from within a program.

$$
\text { system("PERM stim mixed"); }
$$

\section{Randomizing Presentation Order}

PERMUT randomly permutes the elements of its array argument. It takes three arguments: the name of the array to be permuted, the size of the array, and the size of each array element. In this way, the same function can be used to randomize arrays of characters, integers, pointers, and so on. For example, if STIMULUS[I] contains the Ith stimulus, ORDER is an array of integers suitably large, and $I$ is a loop index, instead of using I to access stimulus array elements, as in:

$$
\text { printf(stimulus[i]); }
$$

a permuted ORDER array can be used to randomize presentation order by indirection. In the following example, assume that the array ORDER contains the integers from zero to one less than the number of stimuli, NSTIM. (In C, array indexing begins with zero, not one, as in other languages.)

$$
\begin{gathered}
\text { permut(order, nstim, sizeof (int)); } \\
\ldots \\
\text { printf(stimulus [order }[\mathrm{i}]])
\end{gathered}
$$

The $\mathrm{C}$ primitive, SIZEOF, can be used to determine the size, in bytes, of any data type.

RANDOM is a function that returns randomly selected, uniformly distributed integers. The returned value is between MIN and MAX inclusive, and may be used for presenting randomly selected practice stimuli.

$$
\begin{aligned}
& \text { random(min, max); } \\
& \text { nrandom(min, max }) ;
\end{aligned}
$$

A function related to RANDOM, NRANDOM, returns an integer between min and max inclusive that is normally distributed.

\section{STIMULUS PRESENTATION}

\section{Ordinary Printing}

Stimuli may be printed using standard $\mathrm{C}$ printing procedures; a common one, PRINTF, can print strings in complicated formats, though it is used here in a simple way. For example, the following statement would provide a short debriefing for subjects:

$$
\text { printf("The experiment is over. Thank you."); }
$$

If more efficient stimulus presentation is necessary, the more primitive UNIX function WRITE can be used instead, though the savings are not great for simple strings.

$$
\text { write(1, "The experiment is over. Thank you.",34); }
$$

\section{Screen Printing}

A powerful generalization of PRINTF is PRINTW, which prints at any coordinate on a terminal screen. PRINTW is part of the CURSES screen controlling package developed at UCB (Arnold, Note 1) and uses the TERMCAP data base that contains information on 
terminal's capabilities. CURSES allows programmers to print text at any location on a screen without knowledge of details about individual terminal characteristics. For example, the following program fragment prints a stimulus indented 20 spaces below the 10th line of a clear CRT screen:

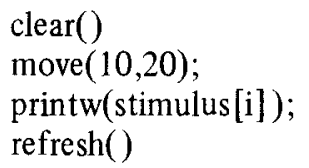

The CLEAR function clears the terminal screen, and the MOVE function moves the cursor to the specified linecolumn coordinates. REFRESH is called after PRINTW to tell CURSES to update the screen. The CURSES package is too large to discuss in this context, but documentation of this well-designed package is available (Arnold, Note 1).

\section{Timed Presentation}

Sometimes stimuli or instructions are to be presented for a fixed duration. This is possible with the UNIX SLEEP function. The following call presents a notice for 60 sec:

$$
\begin{aligned}
& \text { printf(“"Rest for a minute"); } \\
& \text { sleep(60); } \\
& \text { printf(“'Begin Block II"); }
\end{aligned}
$$

In other cases, subjects may be allowed to rest as long as they like, but no more than some maximum duration. An interruptable pause is possible with the CSL TIMEGETC function that uses the UNIX ALARM and SIGNAL functions to wait a specifiable maximum number of seconds for a response. In the following example, TIMEGETC will wait at most 2 min for a keypress to continue:

$$
\begin{aligned}
& \text { printf("Press any key to continue"); } \\
& \text { timegetc(120); } \\
& \text { printf("Begin Block II"); }
\end{aligned}
$$

Both SLEEP and TIMEGETC have a maximum resolution of $1 \mathrm{sec}$, so they are useful only for presentations of greater than a few seconds.

\section{RESPONSE COLLECTION}

\section{Ordinary Response Collection}

One function for reading from the keyboard is the UNIX GETCHAR, which returns one character from the keyboard each time it is called. For example, the follow. ing statement sets response to the next character typed:

$$
\text { response }=\text { getchar }() \text {; }
$$

Whole strings can be read using the UNIX GETS func- tion. GETS obtains a string from the keyboard and stuffs it into its argument. GETS assumes that string is pointing to enough space to hold whatever is input:

$$
\text { gets(string); }
$$

\section{Single-Character Response Collection}

UNIX normally hands characters to functions like GETCHAR and GETS only after a whole line is input and followed by a new-line character (RETURN or LINEFEED). This is called "cooked" mode. In cooked mode, functions like GETCHAR will not allow recording response times for the first character typed, because they wait for a new-line character. Then there is an unread new-line character in an input buffer, and a possibly unwanted character (the one pressed by the responder) on the terminal screen.

The UNIX functions GTTY [get terminal (tty) status] and STTY (set tty status) solve these problems. Rather than describe the obscure syntax of these functions, I will discuss the CSL function, EXPMODE, that uses both. EXPMODE takes one integer argument and puts the terminal into CBREAK and NOECHO modes if it is nonzero (true for $\mathrm{C}$ ) and back to normal mode if it is zero (false for C). In CBREAK mode, in contrast to cooked mode, characters are passed to programs as they are received; new lines are unnecessary. (This mode is new to Version 7 UNIX; the old version counterpart is called "RAW" mode.) EXPMODE also changes the terminal mode so that typed characters are not displayed (NOECHO mode). A side effect of calling EXPMODE is that the input buffer is flushed. This is useful for discarding keypresses made by subjects between trials.

\section{Timed Response Collection}

The UNIX function FTIME allows reading the system clock that ticks at a $60-\mathrm{Hz}$ rate, allowing a maximum resolution of about $17 \mathrm{msec}$. It is used in the CSL PRESENT function, which presents a stimulus and records a timed response not greater than a specified maximum (using TIMEGETC) while in EXPMODE. PRESENT briefly goes into a privileged mode to receive extra CPU attention.

Because UNIX is a timesharing system, the CPU response a program normally gets is inversely proportional to the load of the system. Rather than restrict access to the computer while collecting data, it is possible to increase the priority of a program so that it gets extra CPU cycles. The UNIX function NICE, created to reduce the load of a particular program (to be nicer to other users), can be called with a negative niceness (if the program is owned by the super-user) to receive extra attention. Since other users suffer while a program is negatively niced, use negative niceness for short periods (e.g., while the program is presenting stimuli and timing responses) and return to normal (zero) niceness for other tasks (e.g., randomizing or storing data). 


\section{AN EXPERIMENTAL CONTROL PROGRAM}

A general experimental control program, EXP, uses all the functions discussed so far. To use EXP, no knowledge of its inner workings is required, although it is useful for modification. The main program of EXP uses components for initializing and setting options, presenting instructions, running trials (present stimuli, collect and store data), and cleaning up loose ends. Many of the functions are useful for other programming tasks because they are self-contained; these functions are discussed in following sections.

\section{Initialization}

Initialization involves: (1) setting the values of variables that cannot be set automatically (such as arrays); (2) calling any functions that do further initialization (e.g., the CURSES screen package requires that the functions INITSCR be called); (3) making sure that the program dies gracefully if interrupted (e.g., catching BREAK or DEL characters with the UNIX SIGNAL function); and (4) seeding the random number generator RAND, with SRAND called with the time and date (found with TIME).

\section{Setting Options}

Options can be set by assigning values to variables in an option file read by EXP. A sample option file is shown in Table 1. Each of the variables are described in following sections. EXP gets the name of the option file from a UNIX call like:

\section{EXP options}

These variables are read and interpreted by a set of CSL functions, collectively called VARPAK, that form a standalone variable-handling package. The function READVAR sets the names and values of the variables found in its file argument. If the option file in Table 1 is called "options," the following line sets the variables.

$$
\text { readvar(“options"); }
$$

After variables are set, their values can be determined from names using the GETVAR function. GETVAR returns the string value of the name it is supplied if the

Table 1

Sample Option-Setting File for EXP

$\begin{array}{lll}\text { subjid } & = & \text { subj10 } \\ \text { stimfiles } & = & \text { set1 set2 set3 } \\ \text { randfiles } & = & \text { true } \\ \text { randstim } & = & \text { true } \\ \text { nrepeat } & = & 1 \\ \text { isi } & = & 2 \\ \text { max_rt } & = & 5 \\ \text { clearscreen } & = & \text { true } \\ \text { beep } & = & \text { false } \\ \text { instructfile } & = & \text { instructions } \\ \text { debrieffile } & = & \text { explanation } \\ \text { datafile } & = & \text { data }\end{array}$

name matches that of a set variable; otherwise, it returns a null pointer. Continuing the example with the variables from the option file, the interstimulus interval (ISI) can be set by using the UNIX ATOI (ASCII to integer conversion) function that returns the integer value of a string:

$$
\text { isi = atoi(getvar("isi")); }
$$

Similarly, the name of the file in which data are to be stored can be set.

$$
\text { datafile = getvar(“datafile"); }
$$

Often, it is useful to let variables have truth values, such as "true" or "yes" or some nonzero integer value. For this, the function TRUEVAR is provided. If a variable called varname has any of the values

$$
\text { true yes } 1 \mathrm{t} \text { y (null) }
$$

then

$$
\text { truevar(varname); }
$$

retums one (true in C); otherwise, it returns zero (false in C). This can be used to set an option that has one of two values. For example, the CLEARSCREEN option (to tell EXP to clear the screen between trials) is set to true in the option file in Table 1. This can be used to set an internal variable:

$$
\text { clearscreen = truevar("clearscreen"); }
$$

The VARPAK package also includes functions for interpolating strings containing variables, for setting individual variables, and for printing all the variables set. These functions can simplify setting options and trying many configurations of experimental control programs with EXP.

\section{Presenting Instructions}

For presenting text to subjects, EXP uses the CSL function, SHOWTEXT, that buffers text for the terminal screen. SHOWTEXT takes one argument, the name of the file containing the instructions, and presents text one screen full at a time until the file is exhausted. Subjects pace their reading by pressing a key for each new page. Instructions can be presented with one line when the INSTRUCTFILE variable is set:

$$
\text { showtext(getvar("instructfile")); }
$$

\section{Running Trials and Storing Data}

EXP uses the CSL function, RUNTRIALS, controlling stimulus presentation and data storage. For each stimulus file in the variable STIMFILES (in random order if RANDFILES is true), RUNTRIALS reads all the stimuli and presents them NREPEATS times (in random order if 
RANDSTIM is true). On each trial, the screen is cleared if CLEARSCREEN is true and the program sleeps for the number of seconds to which the variable ISI is set. Finally, the single character response and reaction time are collected by the PRESENT function and are stored in the file named by the variable DATAFILE.

PRESENT takes four arguments:

$$
\text { present(string, beep, rt, max_rt); }
$$

STRING is presented, preceded by a beep if BEEP is nonzero, the reaction time is stored indirectly through the double pointer RT, and the obtained character response is returned by the function. PRESENT returns the character typed unless MAX_RT seconds have passed, in which case it returns zero. PRESENT flushes the input buffer so extra keypresses are discarded, and goes in and out of a privilege mode to get extra CPU attention while waiting for a response (see the earlier discussion of NICE).

\section{Finishing Up}

After all the data are collected, EXP presents to subjects a debriefing it finds in the file named by the variable DEBRIEFFILE. Finally, EXP calls FINISH to clear the screen, say good-by to the subject, and call any functions for normal termination (the CURSES package requires a final call to the ENDWIN function).

\section{Possible Uses of EXP}

EXP can be used as is to run experiments that have several stimuli (perhaps divided into blocks) presented on a clear terminal screen for timed single character responses. This includes simple choice reaction time experiments, and reading and reasoning studies, in which critical timing of stimulus presentation is not needed. For example, a reading experiment might have subjects read a story (each story in its own stimulus file) line by line by pressing the space bar to get successive sentences. Sentence reading times can be recorded and then followed by a last line with some other type of stimulus, perhaps to test comprehension. While the class of paradigms possible with EXP is large, the program is relatively easy to modify.

\section{Interfacing with Data Analysis Programs}

EXP allows easy interfacing with data analysis programs described by Perlman (1980). Data are stored in a file in random order and can easily be reformatted because of the versatile capabilities of data manipulation programs. If data are stored with a stimulus description (e.g., strings coding levels of experimental factors), data can be analyzed immediately after an experiment with no further specification.

\section{RETERENCE NOTE}

1. Arnold, K. C. R. C. Screen updating and cursor movement optimization: A library package. Berkeley, Calif: Department of
Electrical Engineering and Computer Science, University of California, Berkeley, 1980.

\section{REFERENCES}

Jensen, K., \& Winth, N. Pascal user manual and report. New York: Springer-Verlag, 1974.

JoY, W. N. UNIX programmer's manual: Seventh edition (Virtual VAX-11 version). Berkeley, Calif: Department of Electrical Engineering and Computer Sciences, University of California, Berkeley, 1980.

Katzan, H. FORTRAN 77. New York: Van Nostrand Reinhold, 1978.

Kernighan, B. W., \& Richie, D. M. The C programming language. Englewood Cliffs, N.J: Prentice-Hall, 1978.

Perlman, G. Data analysis programs for the UNIX operating system. Behavior Research Methods \& Instrumentation, 1980, 12, 554-558.

Richie, D. M., \& Thompson, K. The UNIX time-sharing system. Communications of the ACM, 1974, 17, 365-375.

Richie, D. M., \& Thom Pson, K. The UNIX time-sharing system. Bell System Technical Journal, 1978, 57, 1905-1929.

\section{NOTE}

1. UNIX is a trademark of Bell Laboratories. DEC, VAX, and PDP are trademarks of Digital Equipment Corporation.

Appendix

Sources and Short Descriptions of Functions

\begin{tabular}{|c|c|c|}
\hline PERM & (CSL) & permut lines in a file \\
\hline system & (Bell) & execute UNIX command at $C$ level \\
\hline permut & (CSL) & permut array elements \\
\hline random & (CSL) & uniform random integer generator \\
\hline nrandom & (CSL) & normal random integer generator \\
\hline printf & (Bell) & print formatted string \\
\hline write & (Bell) & print simple string \\
\hline curses & (UCB) & package of screen printing functions \\
\hline printw & (UCB) & curses version of printf \\
\hline clear & (UCB) & curses screen clearing function \\
\hline move & (UCB) & curses cursor moving function \\
\hline refresh & (UCB) & curses screen updating function \\
\hline sleep & (Bell) & timed suspension of processing \\
\hline timegetc & (CSL) & get a character within time limit \\
\hline alarm & (Bell) & interrupt processing after time limit \\
\hline signal & (Bell) & catch or ignore signals \\
\hline getchar & (Bell) & get a character from keyboard \\
\hline gets & (Bell) & get a string from keyboard \\
\hline gtty & (Bell) & get terminal (tty) status \\
\hline stty & (Bell) & set terminal (tty) status \\
\hline expmode & (CSL) & put terminal in cbreak+noecho mode \\
\hline f time & (Bell) & read the $60-\mathrm{Hz}$ clock \\
\hline present & (CSL) & present stimulus and time response \\
\hline nice & (Bell) & set program priority \\
\hline EXP & (CSL) & general experimental control program \\
\hline initscr & (UCB) & curses initialization function \\
\hline rand & (Bell) & random integer generator \\
\hline srand & (Bell) & seed random integer generator \\
\hline time & (Bell) & get the time and date \\
\hline varpak & (CSL) & package of variable handling functions \\
\hline readvar & $(\mathrm{CSL})$ & varpak: read variables from file \\
\hline getvar & (CSL) & varpak: get a variable's value \\
\hline atoi & (Bell) & ASCII to integer conversion \\
\hline truevar & (CSL) & varpak: return variable's truth value \\
\hline showtext & (CSL) & show textfile buffered for a screen \\
\hline runtrials & (CSL) & basic iteration function \\
\hline endwin & (UCB) & curses termination function \\
\hline
\end{tabular}

(Received for publication September 10, 1981; revision accepted November 23,1981 .) 